\title{
An infant with intradural extramedullary synovial sarcoma: the youngest case in the literature
}

 \\ Alphan Küpesiz ${ }^{1}$, Elif Güler ${ }^{1}$ \\ Departments ${ }^{1}$ Pediatric Hematology and Oncology, ${ }^{2}$ Neurosurgery, ${ }^{3}$ Pathology and ${ }^{4}$ Radiology, Akdeniz University Faculty \\ of Medicine, Antalya, Turkey. E-mail: elifguler@akdeniz.edu.tr \\ Received: 5th November 2018, Revision: 24th December 2018, 13th January 2019, Accepted: 13th February \\ 2019
}

SUMMARY: Yalçın K, Tüysüz G, Kazan S, Gürer EI, Karaali K, Küpesiz A, Güler
E. An infant with intradural extramedullary synovial sarcoma: the youngest case in the literature. Turk J Pediatr 2019; 61: 765-770.

\begin{abstract}
Spinal cord involvement of synovial sarcoma is extremely rare. So far only two cases have been reported. Herein we describe the youngest case in the literature. She is 14-month-old and first presented with difficulty in walking ongoing for a week. Imagining showed a spinal cord mass at C5-T3 levels. The patient had gone under Decompressive surgery and histopathologic examination of the specimen revealed the presence of synovial sarcoma. Although the tumor regressed after chemotherapy, she was lost due to viral pneumonia. Synovial sarcoma should be kept in mind while evaluating spinal tumors even in infantile group.
\end{abstract}

Key words: synovial sarcoma, spinal tumor, pediatric tumor.

Synovial sarcoma (SS) is one of the rare softtissue sarcomas that usually occur in young adults. $^{1,2}$ It can arise in almost at any part of the body, but extremities are the most common sites of primary involvement., 3 SS arising from the spine or metastasizing to the spine are rarely reported in the literature. Almost all of the reported cases are adults and majority of their tumors were located outside the duramater. ${ }^{5,6}$ Here, we present a case of primary intradural extramedullary SS in the cervicothoracic region. To our knowledge, this is the youngest case of intradural extramedullary spinal SS in the literature. ${ }^{7,8}$

\section{Case Report}

A 14-month-old girl presented with difficulty in walking ongoing for a week. Trauma or other predisposing factors were not identified. Neurological examination revealed decreased motor strength (muscle strength: grade 2) and loss of sensation in the lower extremities. She also had tenderness in palpation along the cervical spine. T2 weighted magnetic resonance imaging (MRI) scans of spine indicated an enhancing spinal lesion at C5-T3 levels that compressed the spinal cord. Due to medullary edema on upper and lower spinal segments of the cord, the lesion appeared as intramedullary on sagittal MRI scans (Fig. 1). MRI of thoracal and lomber vertebra also showed leptomeningeal metastases at posterior fossa and conus medullaris (Fig.2). Computed tomographic (CT) scan of the chest revealed metastatic nodules in the left lung.

The patient had gone under decompressive cervicothoracic laminectomy. Macroscopically, the tumor was localized in intradural extramedullary area. A biopsy was obtained in the same procedure. Histological examination of the specimen revealed poorly differentiated SS (Fig. 3) containing intradural necrotic tumoral lesions. Due to macroscopic appearance of the tumor and also pathologic confirmation of dural involvement the patient was diagnosed with intradural extramedullary localized SS. The patient was staged as T1bN0M1 and Group III disease according to the TNM system and 


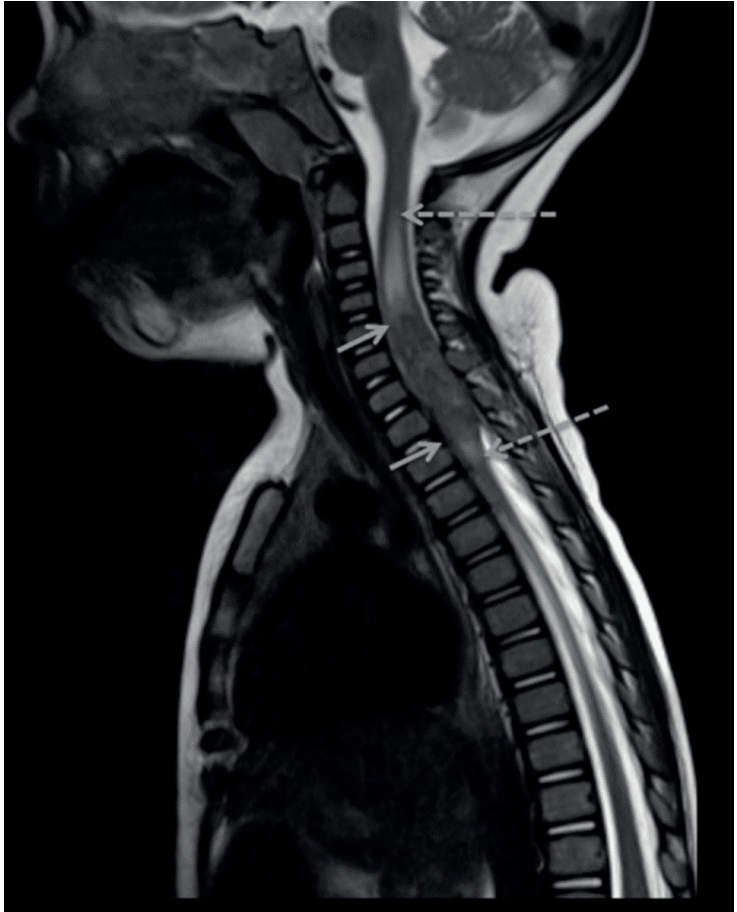

Fig. 1. Magnetic resonance imaging of spinal area. Magnetic resonance imaging (MRI) scan of spine indicated an enhancing extramedullary 6 spinal lesion with the greatest diameter of $3 \mathrm{~cm}$ at $\mathrm{C5}$ T3 levels. This lesion had extended 7 from T1-T2 intervertebral space to left supra clavicular area. There is spinal cord compression 8 at the same level of.

Intergroup Rhabdomyosarcoma study (IRS) respectively. ${ }^{9}$ After recovery from surgery, the patient received two courses of chemotherapy consisting of doxorubicin $\left(30 \mathrm{mg} / \mathrm{m}^{2}\right.$, day $1-2)$, cyclophosphamide (10 mg/kg, day $1-3$ ) and vincristine $\left(1,5 \mathrm{mg} / \mathrm{m}^{2}\right.$, day 1$)$ once every 21 days. After 2 cycles of chemotherapy, the patient neurologically improved (motor strength $4 / 5$ in lower extremities). Spinal MRI demonstrated regression in primary lesion and the metastatic nodules in lung disappeared in the new CT. Unfortunately; the patient was lost due to respiratory failure caused by human parainfluenza virus type 3 pneumonia, two months after diagnosis. Consent from the parents was obtained for the publication of this report.

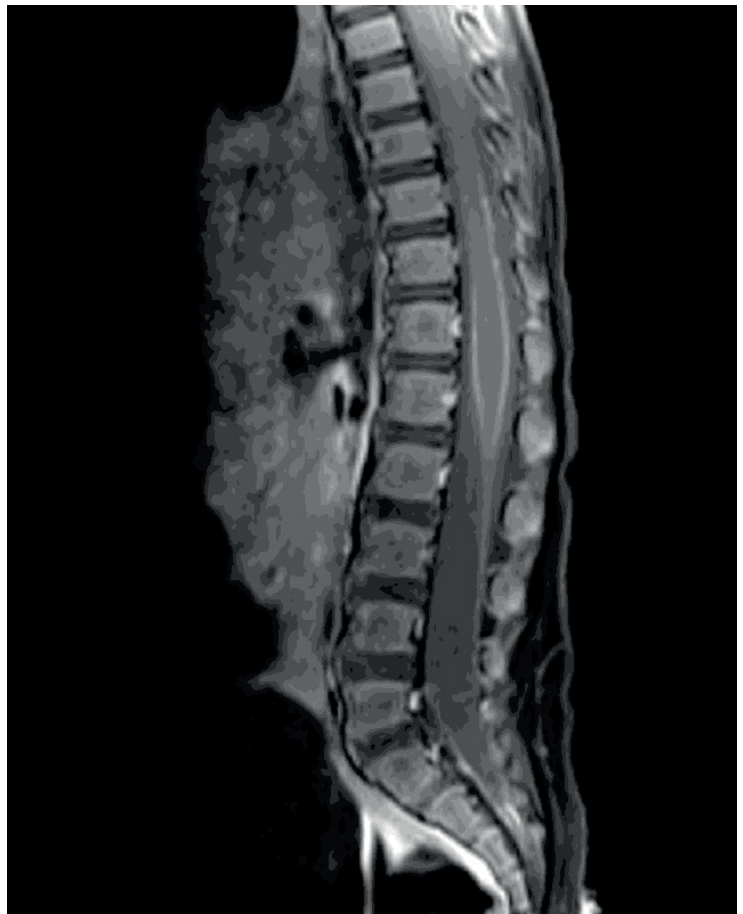

Fig. 2. Magnetic Resonance imaging of thoracal and lomber vertebra.

Magnetic Resonance Imaging of Spine revealed dural contrast enhancements compatible with leptomeningeal metastases in posterior fossa and conus medullaris.

\section{Discussion}

Spinal cord tumors are rare in the pediatric population and they account for $1 \%$ to $10 \%$ of all pediatric central nervous system tumors. ${ }^{10}$ The anatomical classification of the tumors with respect to the dura is; extradural, intradural extramedullary and intradural intramedullary. Intradural extramedullary localization is the rarest form of all. In a retrospective study by Wilson at all, where 35 patients with spinal tumors were evaluated, only $14 \%$ of the spinal tumors were extramedullary located. ${ }^{10}$ Intradural extramedullary tumors are usually leptomeningeal metastases resulting from brain tumors. ${ }^{10}$ If they are primarily originated, they usually are meningeal in origin and include meningiomas and nerve sheath tumors (neurofibromas, schwannomas, ganglioneuromas and malignant nerve sheath tumors). Benign masses like lipoma, vascular lesions, inflammatory disorders, and cystic lesions can also present like a spinal tumor. ${ }^{11}$ Synovial Sarcoma is extremely rare for this 


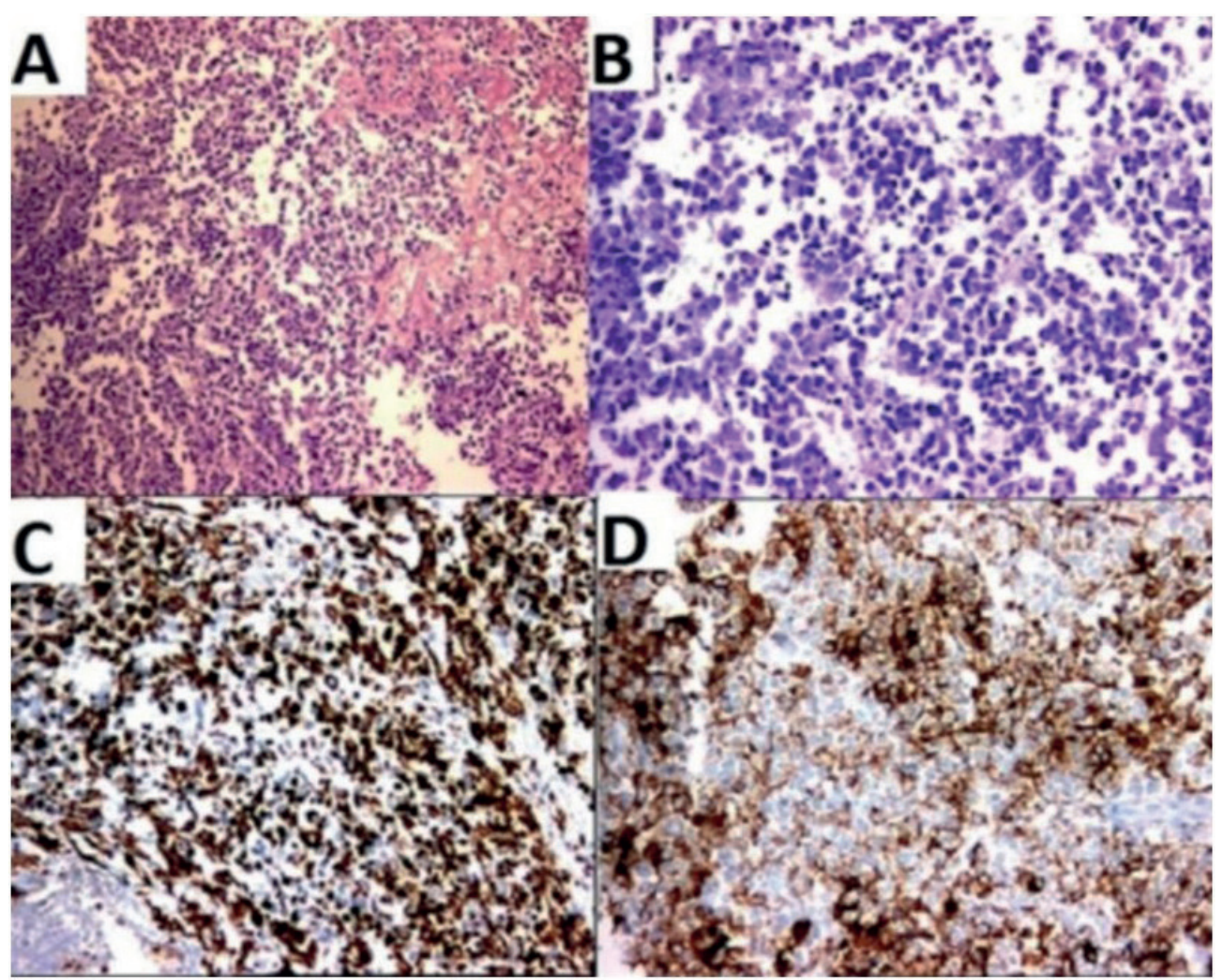

Fig. 3. Histological examination of specimen.

A. Hematoxylin and eosin $(\mathrm{H} \% \mathrm{E})$ staining shows the presence of tumor lessions that form solid irregular islands between wide areas of necrosis. Tumor cell have round nucleus with prominent nucleolus and visible stoplasmic barriers. Tumor cells have numerous mitotic figures, some parts of them are atypical, and they partially form indistinct adenoid like areas. B. Hematoxylin and eosin staining shows Epitheloid pattern $\times$ 100. C. Immunohistochemical staining was positive for Vimentin in stoplasma of tumoral cells $\times 200$. D. Epithelial membrane antigen (EMA) shows membranous staining x 200.

Immunohistochemical staining was positive also for Pan cytokeratin (CK-PAN), CD56, calretinin, CD 34, $\mathrm{Bcl}-2$, and S100. The percentage of Ki-67 positive cells was 50\%.

location. To date, there are only two pediatric reports originating from the intradural extramedullary area. ${ }^{7,8}$ These patients' characteristics are summarized in Table I. Our patient is the third and the youngest among all.

Synovial sarcomas are generally classified as monophasic, biphasic or poorly differentiated. ${ }^{3}$ The latest form is associated with a worse prognosis among all. More than $90 \%$ of all SS show a reciprocal translocation, $t(X$ : 18) (p11:q11), with a resultant fusion gene of SYT and one of three SSX genes. It is considered as the molecular hallmark for the diagnosis of SS in difficult cases. ${ }^{12}$ Our case had poorly undifferentiated synovial sarcoma. Unfortunately, the biopsy specimen was too small to detect translocation in the tumor.

In the presence of spinal cord compression, surgical intervention is mandatory for both decompression and histopathologic identification of the tumor. Complete resection with clear margins remains the most effective treatment for localized SS. ${ }^{13,14}$ Unfortunately, 


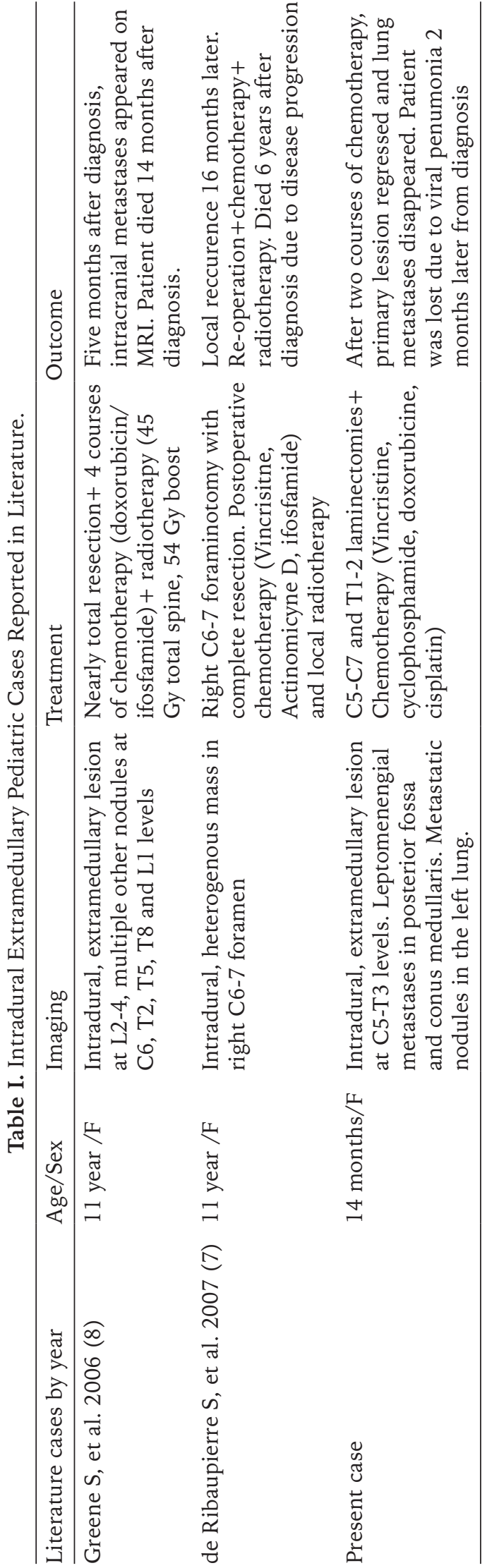

it is difficult to completely remove the lesion because of morbidity in spinal cord localization. Use of adjuvant radiation therapy after surgery provides good local control. ${ }^{15,16}$ If complete surgical resection is not immediately possible, a simple biopsy is recommended, followed by a delayed surgery after tumor reduction with neoadjuvant chemotherapy. ${ }^{17}$ Our case had leptomeningeal and distant metastases at diagnosis. Due to poor localization of the tumor, surgeons could only obtain biopsy from the tumor. The patient was less than 2 years of age, so radiotherapy was postponed. A chemotherapy protocol was initiated and the tumor responded to chemotherapy not only in the primary localization but also in metastatic lesions.

High response rates to chemotherapy in SS have been well documented in pediatric series. Pediatric oncologists tend to consider SS as an intermediate chemo sensitive tumor. ${ }^{4,14,17}$ In children, the response rate to neoadjuvant chemotherapy has been estimated to be between $60-65 \% .4,14,17,18$ Improved outcomes have also been reported in children with adjuvant chemotherapy. Many drugs are active on this tumor, but the most commonly used regimens include doxorubicin and/or ifosfamide. ${ }^{2,14,17}$ In our case, after only two courses of chemotherapy, neurologic examination of the patient dramatically improved and the tumor regressed. Unfortunately, the patient was lost due to viral pneumonia. Even though, we cannot conclude the long-term effect of this chemotherapy, we can suggest using it in the group presenting with non-operable lesions.

Poor prognostic factors for SS include large tumor size $(>5 \mathrm{~cm})$, axial localization, invasiveness, and presence of metastatic disease. Non-extremity tumors were associated with poor event free survival (EFS) and distant recurrence. ${ }^{2,19}$ Spinal SS is a rare entity also in the adult population and up to now, only 18 adult patients have been documented as case reports in the literature. ${ }^{5,6}$ Wide surgical excision with negative surgical margins followed by chemotherapy and/or radiotherapy is the currently recommended treatment in the adult population. To preserve the adjacent structures and limit the neurologic impairment, marginal resection was performed in most of these reported patients. In this 
group of patients, adjuvant radiotherapy and/ or chemotherapy is warranted to obtain remission. Despite treatment, prognosis of spinal SS is poor in the adult population. Even though observation period differs among the reported cases, between the 18 patients with spinal SS reported in the literature, 7 of them died due to disease progression, 4 of them were alive with local $(n: 2)$ or systemic metastases (n:2), 4 of them were alive and free of tumor, and no outcome regarding the follow up was reported for the remaining 3 patients. The 4 patients who are alive with no signs of tumor had gone under marginal resection combined with radiotherapy (in all cases) and chemotherapy (in 2 patients). In our opinion, this shows the importance of multimodal therapy over the survival in the adult population.

The prognosis is also poor in spinal or paraspinal SS in pediatric patients. Most of the reported cases had local or distant metastases and they were lost due to disease progression despite treatment. ${ }^{6,7}$ Two reported pediatric cases in the literature were also lost because of disease progression even though they were operated on and later treated with chemotherapy and radiotherapy.

In summary, we report the youngest case of intradural extramedullary SS. Synovial sarcoma should be kept in mind in the diagnosis of intradural extramedullary tumors at any age. Since it has poor prognosis; multidisciplinary approach and novel agents are necessary to achieve a good long-term outcome.

\section{REFERENCES}

1. Brennan B, Stevens M, Kelsey A, Stiller CA. Synovial sarcoma in childhood and adolescence: a retrospective series of 77 patients registered by the Children's Cancer and Leukaemia Group between 1991 and 2006. Pediatr Blood Cancer 2010; 55: 85-90.

2. Okcu MF, Munsell M, Treuner J, et al. Synovial sarcoma of childhood and adolescence: a multicenter, multivariate analysis of outcome. J Clin Oncol 2003; 21: 1602-1611.

3. Eilber FC, Dry SM. Diagnosis and management of synovial sarcoma. J Surg Oncol 2008; 97: 314-320.
4. Ferrari A, Gronchi A, Casanova M, et al. Synovial sarcoma: a retrospective analysis of 271 patients of all ages treated at a single institution. Cancer 2004; 101: 627-634.

5. Kim J, Lee SH, Choi YL, Bae GE, Kim ES, Eoh W. Synovial sarcoma of the spine: a case involving paraspinal muscle with extensive calcification and the surgical consideration in treatment. Eur Spine J 2014; 23: 27-31.

6. Puffer RC, Daniels DJ, Giannini C, Pichelmann MA, Rose PS, Clarke MJ. Synovial sarcoma of the spine: A report of three cases and review of the literature. Surg Neurol Int 2011; 2: 18.

7. de Ribaupierre S, Vernet O, Beck-Popovic M, Meagher-Villemure K, Rilliet B. Cervical nerve root synovial sarcoma in a child with chromosomal $(\mathrm{X} ; 18)$ translocation. Case report and review of the literature. Pediatr Neurosurg 2007; 43: 382-385.

8. Greene S, Hawkins DS, Rutledge JC, et al. Pediatric intradural extramedullary synovial sarcoma: case report. Neurosurgery 2006; 59: E1339.

9. Maurer HM, Beltangady M, Gehan EA, et al. The Intergroup Rhabdomyosarcoma Study-I. A final report. Cancer 1988; 61: 209-220.

10. Wilson P, Oleszek JL Clayton GH. Pediatric spinal cord tumors and masses. J Spinal Cord Med 2007; 30(Suppl 1): S15-S20.

11. Beall DP, Googe DJ, Emery RL, et al. Extramedullary intradural spinal tumors: a pictorial review. Curr Probl Diagn Radiol 2007; 36: 185-198.

12. Sandberg AA, Bridge JA. Updates on the cytogenetics and molecular genetics of bone and soft tissue tumors. Synovial sarcoma. Cancer Genet Cytogenet 2002; 133: 1-23.

13. Brecht IB, Ferrari A, Int-Veen C, et al. Grosslyresected synovial sarcoma treated by the German and Italian Pediatric Soft Tissue Sarcoma Cooperative Groups: discussion on the role of adjuvant therapies. Pediatr Blood Cancer 2006; 46: 11-17.

14. Ferrari A, De Salvo GL, Brennan B, et al. Synovial sarcoma in children and adolescents: the European Pediatric Soft Tissue Sarcoma Study Group prospective trial (EpSSG NRSTS 2005). Ann Oncol 2015; 26: 567-572.

15. McCarville MB, Spunt SL, Skapek SX, Pappo AS Synovial sarcoma in pediatric patients. AJR Am J Roentgenol 2002; 179: 797-801.

16. Sultan I, Rodriguez-Galindo C, Saab R, Yasir S, Casanova M, Ferrari A. Comparing children and adults with synovial sarcoma in the Surveillance, Epidemiology, and End Results program, 1983 to 2005: an analysis of 1268 patients. Cancer 2009; 115: 3537-3547. 
17. Orbach D, Mc Dowell H, Rey A, Bouvet N, Kelsey A, Stevens MC. Sparing strategy does not compromise prognosis in pediatric localized synovial sarcoma: experience of the International Society of Pediatric Oncology, Malignant Mesenchymal Tumors (SIOPMMT) Working Group. Pediatr Blood Cancer 2011; 57: 1130-1136.

18. Ferrari A, De Salvo GL, Dall'Igna P, et al. Salvage rates and prognostic factors after relapse in children and adolescents with initially localised synovial sarcoma. Eur J Cancer 2012; 48: 3448-3455.

19. Spunt SL, Poquette CA, Hurt YS, et al. Prognostic factors for children and adolescents with surgically resected nonrhabdomyosarcoma soft tissue sarcoma: an analysis of 121 patients treated at St Jude Children's Research Hospital. J Clin Oncol 1999; 17: 3697-3705. 